\title{
大鼠同种异体肝移植急性排异反应的 蛋白质组学研究
}

\author{
张春潮 ${ }^{(1) \dagger}$ 朱 峰 ${ }^{(2)} \dagger$ 尉建锋 $^{(2)}$ 郑树森 ${ }^{(2)}$ 李兰娟 ${ }^{(1)}$
}

(1) 浙江大学医学院附属第一医院传染科, 杭州 310003; (2) 浙江大学医学院附属第一医院外科研究所卫生部多 器官联合移植研究重点实验室, 杭州 310003; (3) 苏州大学附属第三医院肝胆外科, 常州 213003)

\begin{abstract}
摘要为更好的理解原位肝移植免疫排异反应的分子机理, 分别建立了大鼠同种异体肝移植 的动物模型作为急性排异组和大鼠同基因移植的动物模型作为非排异对照组. 利用荧光差异显 示双向凝胶电泳并整合内标法与正、反相荧光标记, 对急性排异组和对照组大鼠肝移植后的肝组 织蛋白质表达谱进行了定量蛋白质组学研究. 结果表明, 有 27 个蛋白点的表达水平在急性排异 组有显著差异, 其中 13 个蛋白点表达水平上调, 与对照组相比比值变化超过 1.5 倍以上, 而 14 个蛋白点表达水平下调, 其相应比值变化超过至少 1.5 倍以上. 19 个差异表达蛋白经胶内酶切后, 利用基质辅助激光解析电离飞行时间质谱获得相应的肽指纹图谱并结合数据库搜索得到鉴定. 这些差异蛋白的分子功能主要表现为氧化还原活性及离子结合活性. 实验结果将有助于进一步

了解器官移植排异反应的分子机理.
\end{abstract}

\section{关键词 蛋白质组学 荧光差异显示双向凝胶电泳 同种异体排异 肝移植}

原位肝移植是目前治疗包括肝硬化、酒精肝及 原发性肝癌等终末期肝病最有效的方法 $[1 \sim 5]$. 虽然不 断提高的外科技术与免疫抑制剂的使用有效提高了移 植的成功率，但移植排异反应仍然是目前临床面临的 一个主要问题, 特别是手术结束后的前几个星期排异 反应发生的可能性仍然很大 ${ }^{[6 ~ 8]}$. 苂光差异显示双向 凝胶电泳是蛋白质组学与代谢组学研究的核心技术, 这一技术能够使我们在同一块凝胶上实现不同样本 蛋白差异表达水平的比较, 从而提供疾病相关的新的 生物标记物信息、疾病相关靶点、疾病发病机理与过 程等有用信息. 因此, 已被广泛应用到癌症以及其它 重要疾病的研究 ${ }^{[9,10]}$. 近几年, 蛋白质组学与代谢组 学开始被应用到器官移植的同种异体排异反应以及
排异反应相关生物标记物的发现等领域 ${ }^{[11]}$. 利用双向 电泳与质谱技术，Pan等人 $[12,13]$ 分析了大鼠原位肝移 植自发性免疫耐受现象并发现了与之相关的一些生 物标记物. Clarke等人 ${ }^{[14]}$ 将尿液蛋白质组学研究应用 于肾移植领域, 并把它发展为一种急性肾移植排异的 诊断方法，显示出这一技术应用于器官移植领域的有 效前景. 本文利用苂光差异显示双向凝胶电泳并结合 基质辅助激光解析电离飞行时间质谱技术，报道大鼠 肝移植急性排异反应全细胞蛋白质组学研究.

\section{1 材料与方法}

\section{1 实验设计与手术方案}

本实验所用大鼠购自北京维通利华公司, 品系 
为近交系雄性Lewis (RT11)和BN (RT1n)大鼠, 8 10 周龄, 体重 $180 \sim 200 \mathrm{~g}$. 实验动物清洁饲养, 术前禁食 $12 \mathrm{~h}$, 自由饮水. 近交系雄性Lewis大鼠作为异基因 移植组供体，近交系雄性BN大鼠作为同基因移植组 供、受体与异基因移植组受体. 将 9 只Lewis大鼠和 27 只BN大鼠分成两组. 第一组：同基因对照组 (从 $\mathrm{BN}$ 大鼠移植到 $\mathrm{BN}$ 大鼠, 9 对); 第二组: 异基因排异组 (从Lewis大鼠移植到BN大鼠，9 对). 手术采用腹腔注 射氯胺酮 $(150 \mathrm{mg} / \mathrm{kg}$ ) 麻醉, 大鼠肝移植模型采用改良 Kamada和Calnel“双袖套法”, 不吻合肝动脉 ${ }^{[15]}$. 手术 均在无菌条件下进行.

\section{2 血清 IL-2 与 IFN- $\gamma$ 水平检测}

抽取对照组与急性排异组肝移植后 BN 大鼠血 清样本. 血清 IL-2 与 IFN- $\gamma$ 水平分别用大鼠 IL-2 (Pierce Biotechnology, Inc. USA)与 IFN- $\gamma$ (BD Biosciences Pharmingen, USA) ELISA 试剂盒测定. 实验 操作均按试剂盒说明书进行.

\section{3 组织切片分析}

移植后的肝组织用 $10 \%$ 福尔马林, $4{ }^{\circ} \mathrm{C}$ 固定过夜, 石蜡包埋, 切片厚度为 $4 \mu \mathrm{m}$, 经 HE 染色后在光学显 微镜下观察其形态学变化.

\section{4 荧光差异显示双向凝胶电泳、凝胶成像与图 像分析}

荧光差异显示双向凝胶电泳根据Alban等人 ${ }^{[16]}$ 报 道的方法进行. 简述如下：从对照组和急性排异组中 分别选取移植后 7 9 天的 6 个受体，并将组内 6 份重 量相等的肝组织相互混合. 向混合后的样品中, 加入 $1 \mathrm{~mL}$ 裂解液(含 $8 \mathrm{~mol} / \mathrm{L}$ Urea, $4 \%$ CHAPS, $30 \mathrm{mmol} / \mathrm{L}$ Tris base), 经双向电泳样品研磨试剂盒(购自美国 Amersham Biosciences公司)充分碾磨、处理样品 5 $\min$ 后， $25000 \times g$ 离心 $20 \mathrm{~min}$ ，小心吸取上清并用 Brandford法测定蛋白浓度. 为了减小误差、提高可信 度，实验采用正、反相荧光标记并整合内标法. 在正 相标记的凝胶中, 取自第一组与第二组的鼠肝总蛋 白 $50 \mu \mathrm{g}$ 分别用 $400 \mathrm{pmol}$ 的荧光染料 Cy3 和Cy5 标记. 而在反相标记的凝胶中, 取自第一组与第二组的鼠 肝总蛋白 $50 \mu \mathrm{g}$ 分别用 $400 \mathrm{pmol}$ 的荧光染料Cy5 和 Cy3 标记. 同时, 取自第一组与第二组的鼠肝总蛋白 各 $25 \mu \mathrm{g}$ 合并后用 $400 \mathrm{pmol}$ 的荧光染料Cy2 标记作为
不同凝胶的内标. 两组荧光染料标记后的样品 根据实验设计等量混合，标记反应用 $1 \mu \mathrm{L} 10 \mathrm{mmol} / \mathrm{L}$ 的赖氨酸终止. 混合后的两组样品又与等体积的 $2 \times$ 裂解缓冲液(含 $8 \mathrm{~mol} / \mathrm{L}$ Urea, $4 \%(w / v)$ CHAPS, 130 $\mathrm{mmol} / \mathrm{L}(\mathrm{w} / \mathrm{v})$ DTT, 2\% Pharmalyte, $\mathrm{pH} 3 \sim 10 \mathrm{~L})$ 混合. 双向电泳前, 含裂解液的样品进一步用水化液(含 8 $\mathrm{mol} / \mathrm{L}$ Urea, 2\% ( $w / v)$ CHAPS, $20 \mathrm{mmol} / \mathrm{L}(w / v)$ DTT, $0.5 \%(v / v)$ IPG buffer pH 3 10 NL, $0.002 \%(w / v)$ bromophenol blue)稀释至 $450 \mu \mathrm{L}$ 终体积. 第一向等电聚 焦采用固相 $\mathrm{pH}$ 梯度凝胶(胶条长 $24 \mathrm{~cm}, \mathrm{pH} 3.0 \sim 10.0$, $\mathrm{NL}$ ), 等电聚焦在 Ettan IPGphor 系统中进行. 第二向 垂直 SDS-PAGE 电泳在 Ettan DALTsix 电泳系统上 进行, 采用 $12.5 \%$ SDS-PAGE 分离. 电泳结束后, 图 像由 Typhoon 9400 凝胶成像仪获得; 用 Decyder DIA 与 BVA 软件完成统计学及蛋白质表达水平相对定量 分析. 与对照组相比, 差异蛋白表达水平比值变化超 过 1.5 倍以上的被认为有意义的差异点并被选取用来 质谱分析. 制备型的凝胶上样 $500 \mu \mathrm{g}$ 并采用银染染 色 ${ }^{[17]}$. 本实验所用双向电泳试剂及相关仪器、软件由 美国Amersham Biosciences公司生产.

\section{5 胶内酶切与 MALDI-TOF 质谱分析}

胶内酶切按我们先前报道的方法进行 ${ }^{[18]}$. 主要 步骤如下: 差异蛋白点从银染后的凝胶中切出并转 入 $1.5 \mathrm{~mL}$ Eppendor管中, 用 $100 \mathrm{mmol} / \mathrm{L} \mathrm{Na}_{2} \mathrm{~S}_{2} \mathrm{O}_{3}$ 和 $30 \mathrm{mmol} / \mathrm{L} \mathrm{K}_{3} \mathrm{Fe}(\mathrm{CN})_{6}(1: 1)$ 对凝胶脱色完全后用 Milli-Q水洗涤 2 次，直到淡黄色基本褪尽. 用 100 $\mathrm{mmol} / \mathrm{L}$ 的 $\mathrm{NH}_{4} \mathrm{HCO}_{3}(\mathrm{pH}$ 8.0)平衡 $20 \mathrm{~min}$ 后用Milli-Q 水洗涤 2 次; 乙腈脱水 2 次后用 SpeedVac真空干燥仪 (Thermo Savant, Holbrook, NY, USA)离心干燥 15 min. 胶内酶切时, 加入 $10 \mu \mathrm{L}$ 测序级胰蛋白酶 $(20 \mathrm{ng} / \mu \mathrm{L}$, 用含 $9 \% \mathrm{ACN}$ 的 $40 \mathrm{mmol} / \mathrm{L} \mathrm{NH}_{4} \mathrm{HCO}_{3}$ 溶解，购自美国 Sigma公司), $37^{\circ} \mathrm{C}$ 过夜消化. 酶解产物用 $50 \mu \mathrm{L}$ 含 $5 \%$ $\mathrm{TFA} / 50 \% \mathrm{ACN}$ 的溶液萃取 2 次. 合并萃取液，经冷冻 干燥仪(Virtis, Gardiner, NY, USA)浓缩至 4 5 $\mu \mathrm{L}$. 酶 切结束后, $1 \mu \mathrm{L}$ 含多肽混合物的样品与 $1 \mu \mathrm{L}$ 含 10 $\mathrm{mg} / \mathrm{mL} \alpha$-氭基-4-差基肉桂酸的溶液(用含 $0.05 \% \mathrm{TFA}$ 和 $50 \% \mathrm{ACN}$ 的溶液配制) 混合点样于质谱分析板上, 空气中自然干燥后用Voyager-DE STR质谱仪(Applied Biosystems, Foster City, CA, USA)进行分析. 采用反 射模式、正离子谱测定、时间延迟提取 $100 \mathrm{~ns}$ ，网格 电压 $63.5 \%$, 离子加速电压 $20000 \mathrm{~V}$, 质谱信号档次扫 
描累加 400 次, 取分子量介于 $1000 \mathrm{Da}$ 到 $4000 \mathrm{Da}$ 的肽峰. 采用标准肽 P14R(单同位素分子 量 $1533.8582 \mathrm{Da}$ )和牛氧化胰岛素 $\mathrm{B}$ 链(单同位素分子 量 $3494.6513 \mathrm{Da}$ )对肽谱进行外标校正, 胰酶自切峰 作为肽谱内标校正. 所得到的 PMF 数据用 Mascot 数 据库(http://www.matrixscience.com/)解析, 搜索条件 如下: 物种: Rodentia /Rodents, 胰酶酶切(允许 1 个漏 切位点), 半胱氨酸碘乙酰化, 质量误差: $100 \mathrm{ppm}$.

\subsection{Western blot 分析}

本实验 Western blot 采用 ECL 检测法，具体操作 过程参考美国 Amersham Biosciences 公司生产的试 剂盒说明书. 简述如下: $30 \mu \mathrm{g}$ 总蛋白从不同组提取 后用 $12 \%$ SDS-PAGE 胶分离, 并转至硝酸纤维素膜. 随后，用含 $5 \%$ 脱脂奶粉的 TBS 溶液封闭硝酸纤维素 膜 $1 \mathrm{~h}$. 封闭结束后, 在摇床上与一抗 $4^{\circ} \mathrm{C}$ 过夜孵浴. 孵浴结束后, 已经结合一抗的硝酸纤维素膜用 TBS 溶液漂洗 3 次, 接着与辣根过氧化酶偶联的二抗溶液 孵浴 $1 \mathrm{~h}$, 实验采用增强化学发光法. Western blot 结 束后的图像由美国 Kodak 公司生产的 Image station 2000R 系统成像, 并用生产商提供的软件进行分析.

\section{2 结果与讨论}

\section{1 受体的生存率与急性排异反应的测定}

我们建立了大鼠同基因肝移植与异基因肝移植 2 组实验模型分别作为对照组与急性排异组. 异基因 移植组的受体平均生存时间为 35 天，而同基因移植 组的受体平均生存时间均超过 100 天. $\mathrm{T}$ 细胞及来自 天然免疫系统的细胞通过非特异性途径包括被 Th1 或 Th2 衍生而来的细胞因子参与的粒性白细胞激活, 它们相互作用共同排斥同种异体移植物 ${ }^{[19]}$. IL-2 与 IFN- $\gamma$ 是Th1 细胞分泌的两个代表性细胞因子, 并且 与移植排异密切相关 ${ }^{[20,21]}$. 与同基因对照组的大鼠 相比，急性排异组的大鼠血清IL-2 与IFN- $\gamma$ 的水平表 达显著增加 $(P<0.05)$, 它们的表达水平在移植后第 7 天达到最高峰(图 1).

组织形态分析也与上述结果基本相同，绝大多 数急性排异发生在移植后 7 15 天. 急性排异组的大 鼠肝脏在移植后 7 天，汇管区有淋巴浸润，在移植后 14 天，淋巴细胞浸润至小叶区并伴有肝实质细胞的 坏死. 与排异组的大鼠不同，长期生存的同基因移植
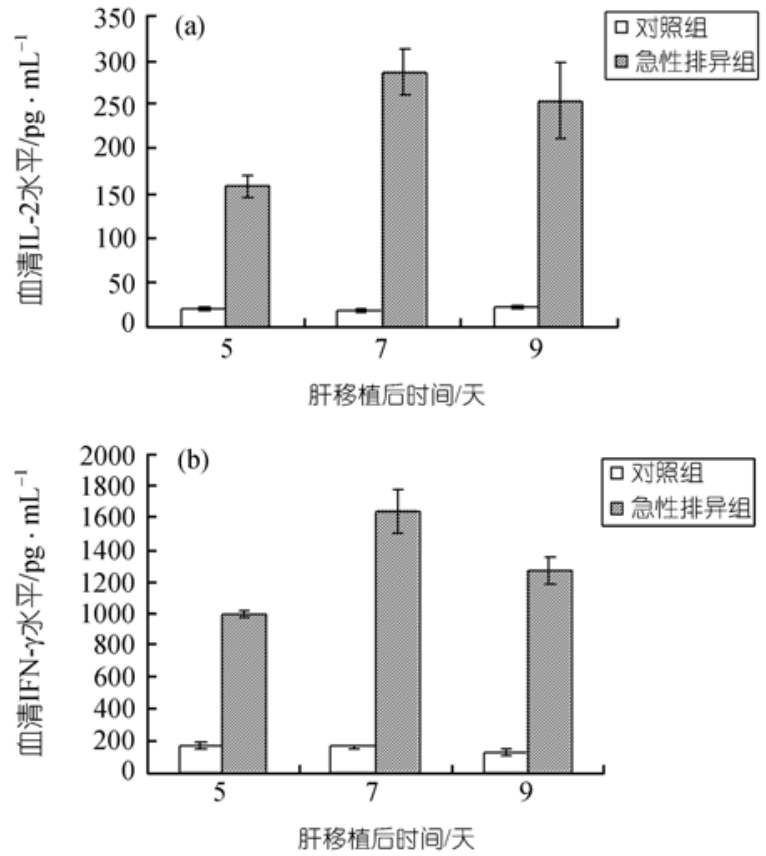

图 1

对照组与急性排异组大鼠肝移植后血清 IL-2(a)与 IFN- $\gamma$ (b)表达水平与 对照组大鼠(空心柱)相比, 急性排异组大鼠(斜线柱) 血清 IL-2 (a)与 $\operatorname{IFN}-\gamma(b)$ 显著增加 $(P<0.05)$, 在移植后 7 天达到最高峰

组的大鼠肝脏组织形态结构完好与正常大鼠的肝脏 形态结构相似(图 2).

\section{2 苂光差异显示双向凝胶电泳与质谱技术分析 异基因移植排异}

由于排异反应绝大多数发生在肝移植后 7 15 天 之间，本实验标本均取自肝移植 7 9 天后的受体，以 此来避免手术后不久严重的炎症反应同时又确保最 佳的排异反应发生时间. 此外，我们采用苂光染料 正、反相标记并整合内标法来提高实验的重复性与 精确的定量效果(图 3).

借助 Decyder-DIA 软件，我们可以从其提供的三 维图像，直观的分析差异蛋白点的表达水平变化. 分 析表明, 有 27 个蛋白点的表达水平在急性排异组发 生显著变化(图 4). 其中, 13 个蛋白点表达水平在急 性排异组中上调，与对照组相比比值变化超过至少 1.5 倍; 而 14 个蛋白点表达水平在急性排异组中下调, 其相应比值变化超过至少 1.5 倍(表 1).

荧光差异显示双向凝胶电泳显示 $\beta$-actin 的表达 水平在(第 20 点)异基因排异组中表达水平上调，与对 照组相比比值为 1.68 倍, 与 Western blot 检测的结果 1.59 倍十分接近(图 5). 在这些差异表达的蛋白点中, 

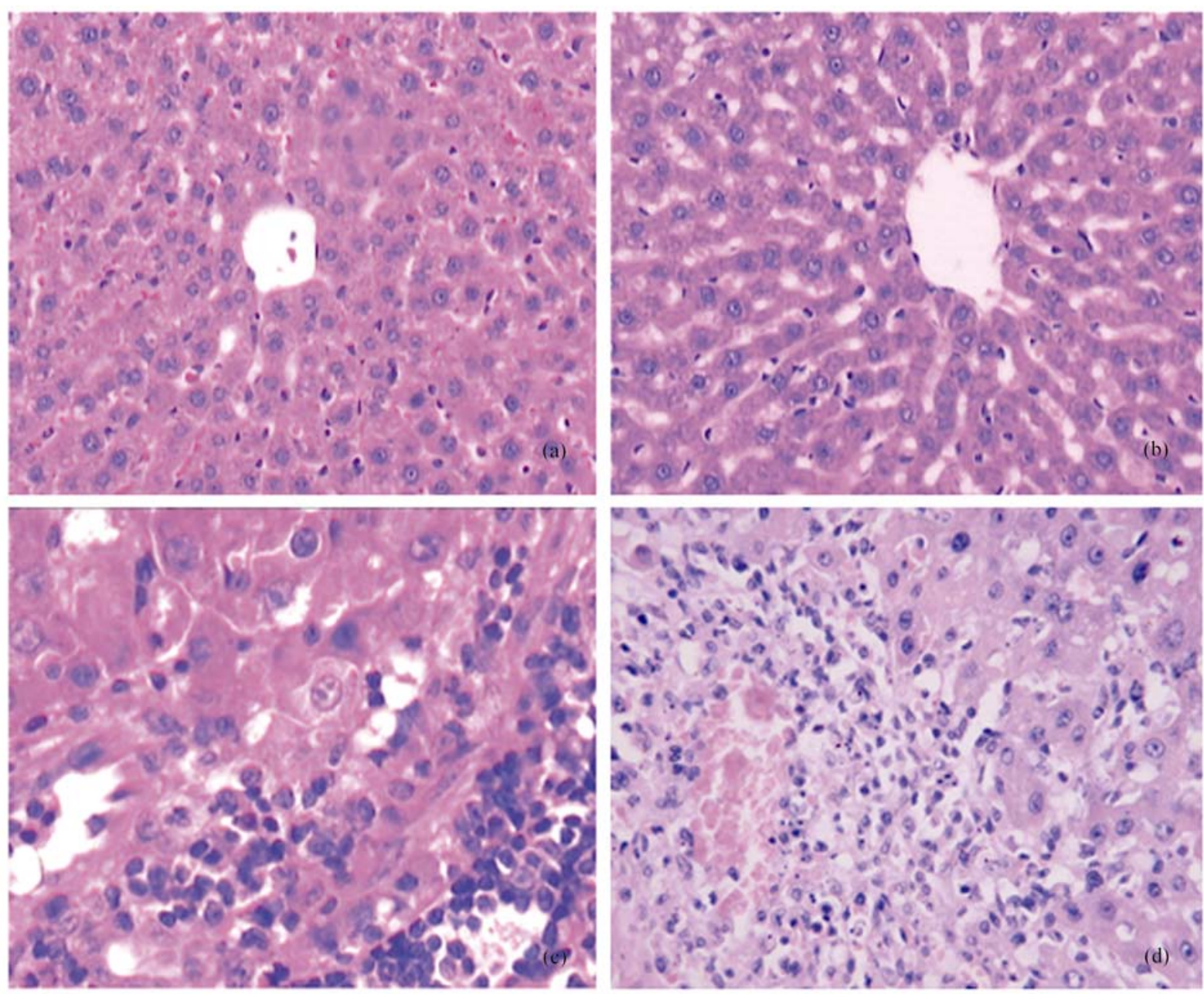

图 2

(a) (d) 组织形态学分析原位肝移植后同基因移植组与异基因移植组急性排异状况移植后的肝组织用 10\%福尔马林固定，石蜡包埋, HE 染色. 对 照组大鼠肝移植后 7 天与 14 天, 肝组织结构形态完好与正常大鼠的肝脏形态结构基本接近((a), (b), HE $\times 200)$. 而急性排异组大鼠肝移植后 7 天, 汇管区较多淋巴细胞浸润. 在移植后 14 天, 淋巴细胞浸润至小叶区并伴有肝实质细胞的大片坏死，中央静脉出现闭塞，静脉内皮炎和胆管内皮炎

19 个蛋白质经胶内酶切、MALDI-TOF质谱分析后，通 过肽指纹图谱得到可靠鉴定. 这些蛋白质执行多种分 子功能, 主要包括氧化还原活性与离子结合活性. 氧 化还原活性与离子结合活性对于正常肝组织的代谢 极为必要与关键. 这些蛋白质的表达水平都出现下降 表明移植后排异组大鼠的肝功能正逐步丧失. 线粒体 乙醛脱氢酶作为一个大分子蛋白主要在细胞质中合 成，然后转运至线粒体基质并加工成一个成熟的酶. 乙醇在肝脏的氧化代谢主要由乙醇脱氢酶与乙醛脱 氢酶催化的. 这两种酶在人类的肝脏中以多种形式存 在，乙醛脱氢酶 II 是清除乙醛的关键酶 ${ }^{[22,23]}$. 乙醛可 能是酒精性肝损伤的的主要物质，乙醛的有害作用 主要包括: 线粒体脂肪酸 $\beta$ 氧化功能的损伤; 氧自由 基的形成; 线粒体谷胱甘肽的缺失. 肝脏中乙醛的浓
度处于其形成与被乙醛脱氢酶降解之间的一个动态 平衡. 乙醛脱氢酶的水平在长期饮酒过度的病人肝 组织中下降并最终导致乙醛在肝脏中的积累. 与对 照组大鼠相比较, 我们的实验表明线粒体乙醛脱氢 酶表达水平在排异组大鼠肝组织损伤时显著下降(比 值变化超过 5.62 倍), 提示这是一个衡量肝损伤与衰 竭的敏感指标. $\beta$-actin是一个细胞骨架蛋白, 对于维 持细胞的结构, 细胞生长极为重要. 最近的研究表明, 细胞骨架的重新组织可能在细胞调亡与坏死中执行 重要功能. 同时，它对于细胞死亡信号传导至线粒体 中也发挥了重要作用 ${ }^{[24]}$. 因此, $\beta$-actin参与独立于 caspase依赖的细胞死亡与坏死. 我们的实验结果与此 相类似, $\beta$-actin在排异组大鼠肝组织损伤与坏死时表 达水平增加，可以部分提示肝功能正在逐步丧失. 

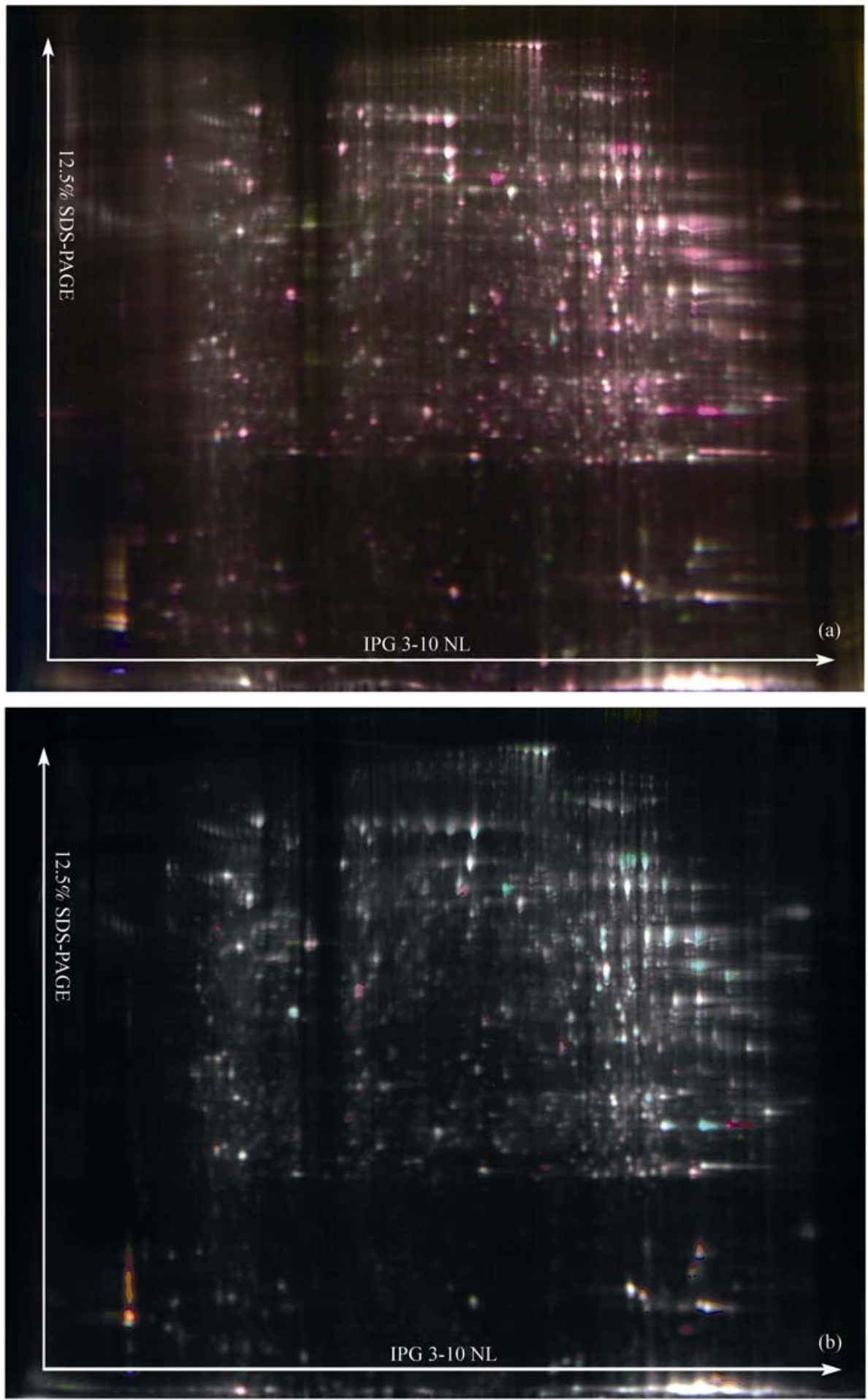

图 3 苂光差异显示双向凝胶电泳整合内标与苂光染料正反相标记法分析异基因原位肝移植大鼠急性排异状况 将同基因移植组与异基因排异组大鼠的肝脏组织分成两组. 在正相标记的凝胶中, 取自第一组与第二组的鼠肝总蛋白 $50 \mu \mathrm{g}$ 分别用苂光染料 Cy3 和 Cy5 标记((a)). 而在反相标记的凝胶中, 取自第一组与第二组的鼠肝总蛋白 $50 \mu \mathrm{g}$ 分别用荧光染料 Cy5 和 Cy3 标记((b)). 而取自第一组与第二 组的鼠肝总蛋白各 $25 \mu \mathrm{g}$ 合并后用荧光染料 Cy2 标记作为不同凝胶的内标((a), (b), 第一向等电聚焦, IPG 3-10, 非线性; 凝胶长度 $24 \mathrm{~cm}$; 第二向 垂直电泳, $12.5 \%$ SDS-PAGE) 
表 1 异基因排异组大鼠肝组织中差异表达水平变化的蛋白点

\begin{tabular}{|c|c|c|c|c|c|c|c|c|}
\hline $\begin{array}{l}\text { 蛋白点 } \\
\text { 序号 }\end{array}$ & 蛋白质名称 ${ }^{a)}$ & $\begin{array}{l}\text { NCBI } \\
\text { 登录号 }\end{array}$ & $\begin{array}{l}\text { 分子量 } \\
\text { /Da }\end{array}$ & 等电点 & $\begin{array}{c}\text { 序列覆盖 } \\
\text { 率/\% }\end{array}$ & $\begin{array}{l}\text { 差异表达 } \\
\text { 水平 }{ }^{b)}\end{array}$ & $t$ - 检验 & 分子功能 ${ }^{c)}$ \\
\hline 1 & $\begin{array}{l}\text { Mitochondrial aldehyde } \\
\text { dehydrogenase precursor }\end{array}$ & gi $\mid 45737866$ & 55566 & 6.69 & $49 \%$ & -5.62 & 0.00022 & Oxidoreductase activity \\
\hline 2 & Sulfite oxidase & gi $\mid 40254758$ & 54320 & 5.86 & $21 \%$ & -3.5 & 0.00051 & $\begin{array}{l}\text { Molybdenum ion } \\
\text { binding }\end{array}$ \\
\hline 3 & Not identified & & & & & -2.7 & 0.00036 & \\
\hline 4 & Catalase & gi|6978607 & 59719 & 7.07 & $35 \%$ & -2.57 & 0.00042 & Oxidoreductase activity \\
\hline 5 & Aconitase 2, mitochondrial & gi|40538860 & 85380 & 7.87 & $15 \%$ & -1.83 & $2.30 \mathrm{E}-06$ & Aconitate hydratase activity \\
\hline 6 & Catalase & gi|6978607 & 59719 & 7.07 & $39 \%$ & -1.74 & 0.0011 & Oxidoreductase activity \\
\hline \multirow[b]{2}{*}{7} & Regucalcin & gi|13928740 & 33368 & 5.27 & $40 \%$ & \multirow[b]{2}{*}{-1.71} & \multirow[b]{2}{*}{$2.40 \mathrm{E}-05$} & Calcium ion binding \\
\hline & $\begin{array}{l}\text { Senescence marker } \\
\text { protein-30 }\end{array}$ & gi| 2507128 & 33367 & 5.40 & $34 \%$ & & & Calcium ion binding \\
\hline 8 & Glutathione S-transferase, mu 2 & gi $\mid 28933457$ & 25686 & 6.90 & $63 \%$ & -1.71 & 0.0031 & $\begin{array}{l}\text { Glutathione transferase activ- } \\
\text { ity }\end{array}$ \\
\hline 9 & $\begin{array}{l}\text { Methylmalonate semialdehyde } \\
\text { dehydrogenase gene }\end{array}$ & gi|13591997 & 57771 & 8.47 & $28 \%$ & -1.66 & 0.001 & Oxidoreductase activity \\
\hline 10 & Peroxiredoxin 4 & gi|16758274 & 30988 & 6.18 & $49 \%$ & -1.63 & 0.0027 & Peroxidase activity \\
\hline 11 & Not identified & & & & & -1.55 & $1.40 \mathrm{E}-05$ & \\
\hline 12 & $\begin{array}{l}\text { Similar to RIKEN cDNA } \\
2300002 \mathrm{G} 02\end{array}$ & gi|34859187 & 49491 & 7.23 & $25 \%$ & -1.54 & 0.00036 & Nucleic acid binding \\
\hline 13 & Not identified & & & & & -1.51 & 0.00045 & \\
\hline 14 & Copine IV & gi|38649068 & 62368 & 5.91 & $24 \%$ & -1.5 & 0.0005 & No annotation \\
\hline 15 & Not identified & & & & & +1.56 & 0.0013 & \\
\hline 16 & Superoxide dismutase 2 & gi|47477896 & 24659 & 8.96 & $29 \%$ & +1.57 & 0.00032 & Manganese ion binding \\
\hline 17 & Not identified & & & & & +1.6 & 0.00074 & \\
\hline 18 & Cofilin 1 & gi $\mid 55778270$ & 18521 & 8.22 & $46 \%$ & +1.63 & $2.10 \mathrm{E}-05$ & Actin binding \\
\hline \multirow{2}{*}{19} & Cyclin D-binding Myb-like protein & gi|34881391 & 60687 & 5.70 & $17 \%$ & \multirow{2}{*}{+1.64} & \multirow{2}{*}{0.0039} & DNA binding \\
\hline & Actin beta & gi|71620 & 41724 & 5.29 & $21 \%$ & & & Motor activity \\
\hline 20 & Actin beta & gi|71620 & 41724 & 5.29 & $33 \%$ & +1.68 & 0.0076 & Motor activity \\
\hline 21 & Not identified & & & & & +1.8 & 0.0002 & \\
\hline 22 & $\begin{array}{l}\text { Similar to purine-nucleoside } \\
\text { phosphorylase }\end{array}$ & gi|34869683 & 32281 & 6.46 & $41 \%$ & +1.86 & 0.00065 & Transferase activity \\
\hline 23 & Diacylglycerol O-acyltransferase 2 & gi|21214749 & 16656 & 9.58 & $42 \%$ & +2.06 & 0.00084 & Acyltransferase activity \\
\hline 24 & Not identified & & & & & +2.22 & $2.90 \mathrm{E}-06$ & \\
\hline 25 & Actin beta & gi|71620 & 41724 & 5.29 & $24 \%$ & +2.3 & $9.00 \mathrm{E}-05$ & Motor activity \\
\hline 26 & Actin beta & gi|71620 & 41724 & 5.29 & $30 \%$ & +1.59 & 0.0014 & Motor activity \\
\hline 27 & Not identified & & & & & +1.73 & 0.00062 & \\
\hline
\end{tabular}

a) 用 MASCOT 库鉴定的蛋白质分值均十分显著 $(P<0.05)$; b) 加、减符号分别表示差异蛋白表达水平在异基因排异组大鼠肝组织中增加与 减少; c) 蛋白质功能分类基于 GOblet 序列分析(http://goblet.molgen.mpg.de/cgi-bin/goblet-batch.cgi) 


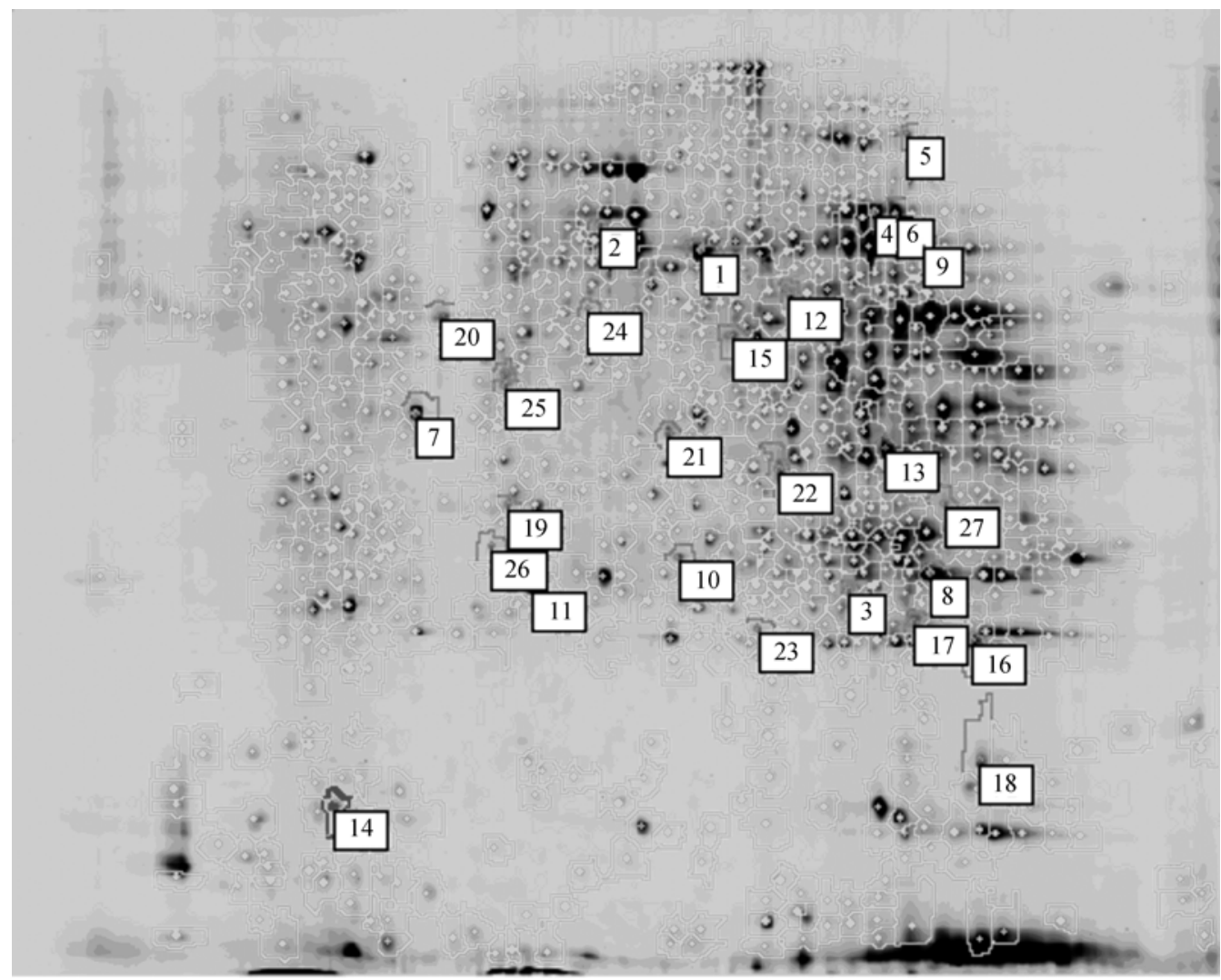

图 4 Decyder-DIA 软件分析差异蛋白表达水平

与对照组相比, 27 个蛋白点的表达水平在急性排异组发生显著变化. 其中, 13 个蛋白点表达水平上调, 与对照组相比比值变化超过 1.5 倍 而 14 个蛋白点表达水平下调, 相应比值变化超过 1.5 倍
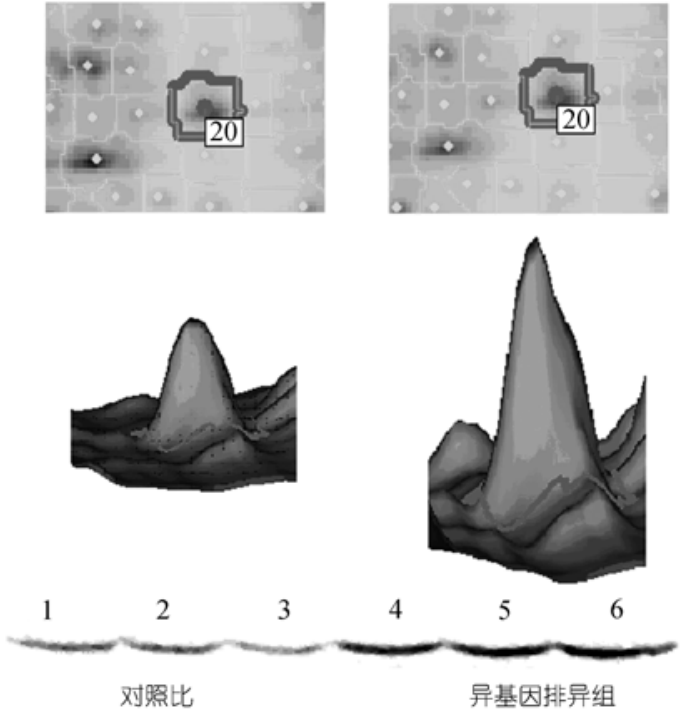

图 5 苂光差异显示双向凝胶电泳与 Western blot 分析蛋白质 差异表达水平结果比较

苂光差异显示双向凝胶电泳显示 $\beta$-actin 的表达水平在异基因排异 组中表达水平增加 1.68 倍(第二组), 与 Western blot 检测的结果增加 1.59 倍十分接近

\section{3 结论}

荧光差异显示双向凝胶电泳与质谱技术的联用 是研究器官移植的有效手段. 本文的部分实验结果 可以帮助我们更好的了解肝移植排异反应的分子机 理，同时也有助于我们发现一些新的与移植排异及 衡量肝衰竭相关的生物标记物.

致谢 作者衷心感谢 Marko 博士对论文的修改与建 议.

\section{参考文献}

1 Curry M P. Hepatitis B and hepatitis C viruses in liver transplantation. Transplantation, 2004, 78(7): 955-963[DOI]

2 Anantharaju A, Van Thiel D H. Liver transplantation for alcoholic liver disease. Alcohol Res Health, 2003, 27(3): 257-268

3 Wong L L. Current status of liver transplantation for hepatocellular cancer. Am J Surg, 2002, 183(3): 309-316[DOI]

4 Frilling A, Malago M, Broelsch C E. Current status of liver transplantation for treatment of hepatocellular carcinoma. Dig Dis, 2001, 19(4): 333-337[DOI]

5 Saadeh S, Davis G L. Management of ascites in patients with 
end-stage liver disease. Rev Gastroenterol Disord, 2004, 4(4): 175185

6 Gomez-Manero N, Herrero J I, Quiroga J, et al. Prognostic model for early acute rejection after liver transplantation. Liver Transpl, 2001, 7(3): 246-254[DOI]

7 Neuberger J. Incidence, timing, and risk factors for acute and chronic rejection. Liver Transpl Surg, 1999, 5(4): S30-36

8 Janssen H, Lange R, Erhard J, et al. Serum bile acids in liver transplantation early indicator for acute rejection and monitor for antirejection therapy. Transpl Int, 2001, 14(6): 429-437[DOI]

9 Zhou G, Li H, DeCamp D, et al. 2D differential in-gel electrophoresis for the identification of esophageal scans cell cancer-specific protein markers. Mol Cell Proteomics, 2002, 1(2): 117-124[DOI]

10 Seike M, Kondo T, Fujii K, et al. Proteomic signature of human cancer cells. Proteomics, 2004, 4(9): 2776-2788[DOI]

11 Wishart D S. Metabolomics: the principles and potential applications to transplantation. Am J Transplant, 2005, 5(12): 2814$2820[\mathrm{DOI}]$

12 Pan T L, Wang P W, Huang C C, et al. Expression, by functional proteomics, of spontaneous tolerance in rat orthotopic liver transplantation. Immunology, 2004, 113(1): 57-64[DOI]

13 Pan T L, Goto S, Lord R, et al. Proteome analysis in liver transplantation. Transplant Proc, 2001, 33(1-2): 156[DOI]

14 Clarke W, Silverman B C, Zhang Z, et al. Characterization of renal allograft rejection by urinary proteomic analysis. Ann Surg, 2003, 237(5): 660-665[DOI]

15 Kamada N, Calne R Y. A surgical experience with five hundred thirty liver transplants in the rat. Surgery, 1983, 93(1 Pt 1): 64-69

16 Alban A, David S O, Bjorkesten L, et al. A novel experimental design for comparative two-dimensional gel analysis: Two-dimen- sional difference gel electrophoresis incorporating a pooled internal standard. Proteomics, 2003, 3(1): 36-44[DOI]

17 Yan J X, Wait R, Berkelman T, et al. A modified silver staining protocol for visualization of proteins compatible with matrix-assisted laser desorption/ionization and electrospray ionization-mass spectrometry. Electrophoresis, 2000, 21(17): 36663672[DOI]

18 Zhang C, Wei J, Zheng Z, et al. Proteomic analysis of Deinococcus radiodurans recovering from gamma-irradiation. Proteomics, 2005, 5(1): 138-143[DOI]

19 Le Moine A, Goldman M, Abramowicz D. Multiple pathways to allograft rejection. Transplantation, 2002, 73 (9): 1373-1381[DOI]

20 Rogers L A, Zlotnik A, Lee F, et al. The maintenance of lytic specificity during the development of clones of cytotoxic $\mathrm{T}$ lymphocytes from single precursor cells. J Immunol Methods, 1991, 143(2): $241-250[\mathrm{DOI}]$

21 Kaminski E R, Kaminski A, Bending M R, et al. In vitro cytokine profiles and their relevance to rejection following renal transplantation. Transplantation, 1995, 60(7): 703-706[DOI]

22 Bosron W F, Li T K. Genetic polymorphism of human liver alcohol and aldehyde dehydrogenases and their relationship to alcohol metabolism and alcoholism. Hepatology, 1986, 6(3): 502-510

23 Yokoyama A, Kato H, Yokoyama T, et al. Genetic polymorphisms of alcohol and aldehyde dehydrogenases and glutathione S-transferase M1 and drinking, smoking, and diet in Japanese men with esophageal squamous cell carcinoma. Carcinogenesis, 2002, 23(11): 1851-1859[DOI]

24 Li J, Li Q, Xie C, et al. $\beta$-actin is required for mitochondria clustering and ROS generation in TNF-induced, caspase-independent cell death. J Cell Sci, 2004, 117(20): 4673-4680[DOI] 